\title{
Stochastic Gradient Approach for Energy and Supply Optimisation in Water Systems Management
}

\author{
A.A. Gaivoronski ${ }^{a}, \underline{\text { J. Napolitano }}^{b}$ and G.M. Sechi $^{b}$ \\ a Department of Industrial Economics and Technology Management, NTNU, Trondheim, Norway \\ ${ }^{b}$ Department of Civil and Environmental and Architecture, University of Cagliari, Cagliari, Italy \\ Email: jacopo.napolitano@unica.it
}

\begin{abstract}
Under conditions of water scarcity, energy saving in operation of water pumping plants and the minimisation of water deficit for users and activities are frequently contrasting requirements, which should be considered when optimising large-scale multi-reservoirs and multi-users water supply systems. Undoubtedly, a high uncertainty level in predicted water resources due to hydrologic input variability and water demand behaviour characterizes this problem.

The aim of this paper is to provide an efficient decision support system considering emergency water pumping plants activation schedules. The obtained results should allow the water system's authority to adopt a robust decision policy, minimising the risk of harmful future decisions concerning the water resource management. The model has been here developed to manage this problem, in order to reduce the damages due to shortage of water and the energy-cost requirements of pumping plants. Particularly, in optimisation, we look for optimal rules considering both historical and generated synthetic scenarios of hydrologic inputs to reservoirs. Hence, using synthetic series, we can analyse climate change impacts and optimise the activation rules considering future hydrologic occurrences.

A simulation model has been coupled with an optimization module using the stochastic gradient method to get robust pumping activation thresholds. This method allows to solve complex problems, solving efficiently large size real cases due to high number of data and variables. Thresholds values are identified in terms of critical storage levels in supply-reservoirs.

Application of the modelling approach has been developed on a real case study in a water-shortage prone area in south-Sardinia (Italy), characterized by Mediterranean climate and high annual variability in hydrological input to reservoirs. By applying the combined simulation procedure, a robust decision strategy in pumping activation was obtained. Developing the stochastic gradient model, a main programming supports has been built by MATLAB efficiently interfaced with CPLEX for optimisation and Excel for inputs and results representation.
\end{abstract}

Keywords: Energy and water supply optimisation, water pumping schedules optimisation, stochastic gradient method 


\section{INTRODUCTION}

Energy saving in operation of water pumping plants and reduction of water deficit for users are frequently conflicting issues when optimising large-scale multi-reservoirs and multi-users water supply systems. Undoubtedly, a high uncertainty level due to hydrologic input variability and water demand behaviour characterizes this problem. The optimisation of water supply systems under a high uncertainty level has been a subject of interest in the research literature (Cunha and Sousa, 2010; Kang and Lansey, 2014; Yuan et al., 2016). In this context, pumping plants' activation schedules are a relevant topic recently treated in Napolitano et al. (2016), considering complex water resource systems management. The successful approaches to solve these kind of problems try to incorporate the uncertainties in the management of water systems by searching for a robust decision policy. The aim is to reach a decision support system, in order to achieve a decision policy that minimises the risk of making wrong future decisions. Consequently, optimisation results should provide the water system authority with optimal rules considering a large set of generated synthetic scenarios of hydrologic inputs to reservoirs. Using synthetic series it is possible to take into account the climate change impacts and balance the rules while also considering future behaviour under the risk of the occurrence of shortages and the cost of early warning procedures to avoid water scarcity, mainly related to activation of emergency water transfers. Thereafter, this problem has been faced considering an efficient optimisation tool based on the Stochastic Gradient method (Gaivoronski, 2005), which is able to encounter considerable problems and manage large-size real cases, due to the high number of data and variables in scenarios.

\section{OPTIMISATION UNDER UNCERTAINTY AND STOCHASTIC GRADIENT METHODS}

The main aim of this paper is to define the optimal thresholds for the activation of the pumping stations. When the reservoirs' storage volumes cross these thresholds, the activation of pumping is triggered. In order to optimise the activation rules, a critical stored volume in reservoirs that may supply the downstream demand centres should be defined. To compose the multi-period model, it is necessary to define a multi-period network starting from a basic graph given by a single-period static design of the water system. The multi-period network is constructed by repeating this single period model for every period and connecting replies using specific rules, as described in Sechi and Zuddas (2008).

There is a need for considering different hydrological scenarios derived by climate change effects in the Mediterranean area. The effect in the optimisation model is an increment in complexity due to time-horizon dimensions: the dimensions of the multi-period model become sizable and it is very difficult to manage and efficiently solve this stochastic problem. In particular, high number of linear and nonlinear variables and parameters are involved managing these kind of models. To make them efficient, we need special algorithms, designed for optimization under uncertainty: therefore, the Stochastic Gradient method (Birge and Louveraux, 2001; Gaivoronski, 2005) is used here as an efficient tool.

This approach belongs to a class of methods specifically designed for problems with continuous distributions of random parameters and nonlinear optimization problems. These methods are suited for optimisation and simulation models, where an analytical relationship between the objective function and parameters is difficult to obtain. Supply chain management, energy generation, and financial applications are some areas of research where these approaches have been successfully used (Gaivoronski, 2005).

The stochastic quasi-gradient (SQG) could be described as the statistical estimate of the gradient of the objective function, which provides an optimal direction for iterative updating of the current approximation to the solution of the optimisation problem.

These methods solve the stochastic optimisation problems of the following type:

$$
\underset{x \in X}{\operatorname{Minimize}} \mathbb{E}_{\omega} f_{0}(x, \omega)
$$

where $\mathbb{E}_{\omega}$ is an expected value considering $\omega \in \mathbb{R}^{k}$ as a vector of random parameters and $x \in X \subseteq \mathbb{R}^{n}$ is a vector of decision variables; $X$ represents the set of feasible solutions.

The optimisation algorithm starts from an initial point $x^{0}$ and moves forward to the current approximation $x^{s}$ of the optimal solution of the original problem (1) according to the following rule:

$$
x^{s+1}=\pi_{X}\left(x^{s}-\rho_{s} \xi^{s}\right)
$$

where $\rho_{s}$ represents the size of the step in the direction opposite to the current estimate $\xi^{s}$ of the gradient $F_{0}(x)$ at the point $x^{s}$. The resulting point is projected onto the set X. The projection operator $\pi_{X}$ transforms an arbitrary point $z \in \mathbb{R}^{n}$ into the point $\pi_{X}(z) \in X$ such that: 


$$
\left\|z-\pi_{X}(z)\right\|=\min _{x \in X}\|z-x\|
$$

the structure of the set $X$ should allow a fast solution of the problem (3); indeed, it should be solved many times during the optimisation. This means that the set $X$ should be defined by linear constraints in order to guarantee an efficient solution process.

The crucial part of the SQG implementation consists in the evaluation of statistical estimates $\xi^{s}$ of the gradient of the objective function $F_{0}(x)=\mathbb{E}_{\omega} f_{0}(x, \omega)$ in (1). This estimate is characterized by considerable flexibility since it is sufficient that the following condition is satisfied:

$$
\mathbb{E}\left(\xi^{s} \mid \mathbb{B}_{s}\right)=F_{0 x}\left(x^{s}\right)+a_{s}
$$

here, $\mathbb{B}_{s}$ is the $\sigma$-field, defined by the history of the process, while $F_{0 x}\left(x^{s}\right)$ represents the gradient of the function $F_{0}(x)$ at the point $x^{s}$. The vector $\xi^{s}$, which satisfies the property (4), is called the Stochastic Gradient. For example, in the simplest case, the stochastic gradient $\xi^{s}$ can be obtained as follows:

$$
\xi^{s}=f_{0 x}\left(\omega^{s}, x^{s}\right)
$$

where $\omega^{s}$ is a single observation of a random vector $\omega$. When the classical gradient does not exist, such as with convex but non-smooth functions, $\xi^{s}$ can be estimated through a generalization of the gradient called the stochastic quasi-gradient $(S Q G)$.

The step-size definition is another fundamental aspect when using the SQG algorithm. Indeed, the convergence to the optimal solution depends on the selection of the correct step $\rho_{s}$, which should satisfy the following property:

$$
\rho_{s} \geq 0, \quad \rho_{s} \rightarrow 0 \quad \sum_{s=0}^{\infty} \rho_{s}=\infty
$$

The required condition in (6) that the step-size tends to zero is not necessary if the precision of the stochastic gradient $\xi^{s}$ increases with iterations. On the other hand, $\rho_{s}$ tends to zero faster if the variance of the stochastic gradient grows with the number of iterations. A recursive simulation and optimisation process can efficiently optimise the simulation models by applying the SQG algorithm iteratively. This methodology is suited for optimisation problems characterized by a large number of decision variables, described by a simulation model for each single problem. Herein, the SQG model has been applied in order to face a real problem of water resource management that is characterized by several decision variables and an extended time horizon needed in the optimisation.

\section{SQG SOLVING A WATER RESOURCE MANAGEMENT PROBLEM}

We consider a water management problem aiming to minimise the pumping energy and management costs needed in order to avoid water deficits for users. This problem has been detailed in previous papers (Gaivoronski et al., 2012; Napolitano et al., 2016). The SQG optimization approach tries to obtain a robust decision policy, minimising the risk of taking wrong and harmful decisions for the future. We assume that the resource in question is scarce and that, for this reason, demands cannot be satisfied in many scenarios. In such situations of scarcity, managers should develop an emergency policy to alleviate the effect of shortages. In order to do so, each user should know in advance the reduced target level of demand satisfaction that the system manager is willing to deliver to him. This target value has to be "barycentric" relative to future uncertain hydrological scenarios. In this way, we can also manage the activation of some emergency measures, such as decreasing the water distributed to the users or activating emergency transfers.

Specifically, we consider finding the optimal set of parameters $q$ that describe the activation pumping rules while minimising the average monthly costs, which are the sums of all costs supported in the water system management. The network state $v^{t}$ (water volumes in reservoirs) evolves in discrete time $t=1, \ldots, T$ (months). At each $t$, water demand $d^{t}$ and inflow $r^{t}$ arrives. Therefore, the pumping schedules are defined by pumping rules with parameters $q$, and at each $t$; the network flows $x^{t}$ are obtained by minimisation of costs (7):

$$
C^{T}\left(q, v^{t}, d^{t}, r^{t}\right)=\min _{x \in X} C\left(x, q, v^{t}, d^{t}, r^{t}\right)
$$

subject to constraints (8) (flow continuity, bounds, etc.).

$$
\Phi\left(x, q, v^{t}, d^{t}, r^{t}\right)=0
$$

The state $v^{t+1}$ at the beginning of period $t+1$ is obtained from the state equation (9): 


$$
v^{t+1}=\Psi\left(x^{t}, q, v^{t}, d^{t}, r^{t}\right)
$$

where the functions $C(\cdot), \Phi(\cdot)$, and $\Psi(\cdot)$ are linear with respect to $(x, v)$.

The objective is to find the set of parameters $q=\left(q_{1}, \ldots, q_{n}\right)$ that minimises the average steady state costs, thus solving the optimisation problem with an infinite time horizon (10).

$$
\underset{q \in Q}{\operatorname{Minimize}} F(Q), F(q)=\lim _{t \rightarrow \infty} \frac{1}{t} C^{t}\left(q, v^{t}, d^{t}, t^{t}\right)
$$

where $Q$ is some feasible set for parameters $q$.

This problem has been solved using SQG methods described in the previous section; the solution approach resorts to a concurrent interrelation between simulation, optimisation, and evaluation steps. In the following, we give a description of the concurrent interaction between them:

- Simulation step for all threads with LP searching for optimal flows: the simulation process is referred to each single period $t$ of the time horizon and is characterized by n-processes simultaneously. Each process has different sets of pumping activation threshold parameters $q$ according to (11):

$$
q^{t}+\delta e_{k}
$$

where $\delta>0$ is a small positive value and $e_{k}$ is a vector of zeros with value 1 in the k-th position. Here the objective function of costs referred to the single period $t$ (7) is minimized in order to obtain an optimal configuration of the network water flows $x^{t}$.

- Optimisation step searching for stochastic gradients: the optimization process will be applied between two consecutive periods and the new parameter configuration will be evaluated according to equation (12):

$$
q^{t+1}=\prod_{Q}\left(q^{t}-\rho_{t} \xi^{t}\right)
$$

where $\rho_{t}>0$ is the step size and $\prod_{Q}(\cdot)=\left(\xi_{1}^{t}, \ldots, \xi_{n}^{t}\right)$ will be estimated by (13):

$$
\xi_{k}^{t}=\frac{C_{k}^{t}-C_{0}^{t}}{\delta}
$$

- Evaluation step: simultaneously, there is an estimation step based on a moving average.

$$
C_{0}^{t+1}=\left(1-\alpha_{t}\right) \bar{C}_{0}^{t}+\alpha_{t} C_{0}^{t}
$$

The estimated costs $C_{0}^{t+1}$ are dependent on the average costs $\bar{C}_{0}^{t}$, referred to all periods, and the costs evaluated in the previous step $C_{0}^{t}$. The relationship between these terms is regulated by an averaging parameter $\alpha_{t}$.

\section{CASE STUDY: SOUTHERN SARDINIA WATER SUPPLY SYSTEM}

In order to test its effectiveness, the SQG approach has been applied to a real case study of the water supply system of southern Sardinia (Italy), which is shown in Figure 1, considering the pumping energy needed in order to avoid the water deficits documented by different hydrological series.

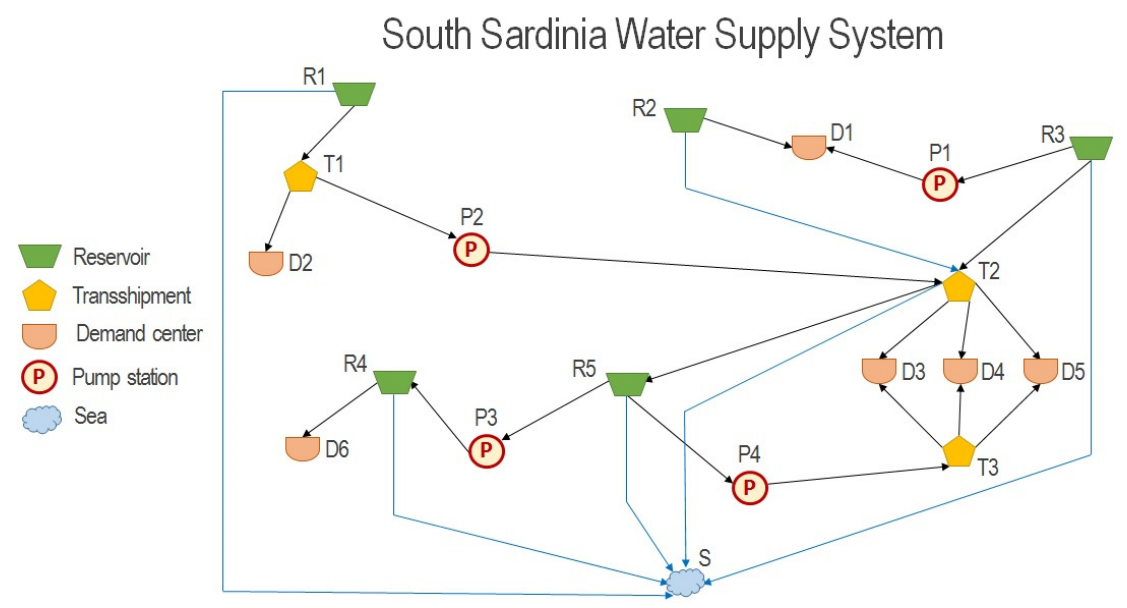

Figure 1. The south Sardinia water supply system 
Water sources are given by five artificial reservoirs, while the water demands have been grouped in six centres according to three different uses: civil, irrigation, and industrial. Pump stations allow demand centres to be supplied with increased economic burden, namely by incurring pumping costs in addition to the ordinary management costs. All the features of this system have been extensively described in Napolitano et al. (2016).

Hydrological inputs refers to observed data reported in the Sardinia-Region Water Plan (RAS, 2006). Synthetic hydrological inflows to reservoirs have been generated starting from this historical database by a Monte Carlo procedure. More information about this generation procedure can be found in Napolitano et al. (2016).

Taking into account this synthetic database, the time horizon for optimisation has been expanded to 6360 monthly periods.

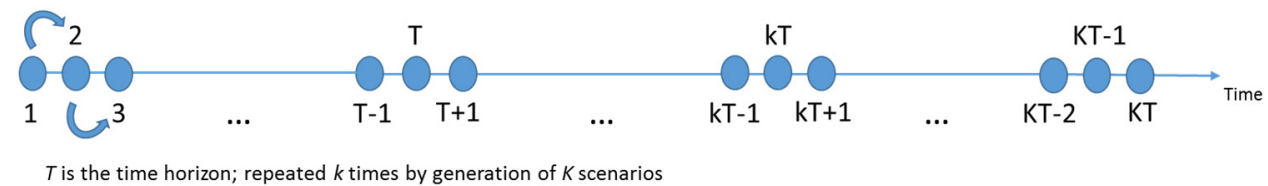

Figure 2. SQG hydrologic inputs organization

As shown in Figure 2, for each reservoir the monthly water inputs are organized in a unique database by arranging the hydrological scenarios consecutively. This data organization is drafted in Figure 2 and allows dealing efficiently with the parallel computational processes necessary in the SQG implementation. The quality of the final solution is strictly related to the length of the hydrological time horizon and to the number of performed iterations.

\subsection{Recursive simulation and optimisation process}

The SQG method approaches this problem by a current simulation and optimisation, as previously described. The SQG model was implemented in MATLAB, interfaced with CPLEX as the optimisation engine and with Excel being used for inputs and results representation.

The final result is a set of activation threshold values and a final configuration $\theta^{t}$ of the water flows along the network's links, which are dependent on the selection of parameters $q$ and system state $v^{t}$.

$$
\Theta^{t}=f\left(q, v^{t}\right)
$$

The set of optimised activation thresholds for pumping transfers, obtained using SQG and the extended hydrology, are reported in Table 1.

Table 1. Optimised activation thresholds values

\begin{tabular}{lllll}
\hline $\begin{array}{l}\text { Activation } \\
\text { Threshold }\end{array}$ & $\mathrm{S} 1$ & $\mathrm{~S} 2$ & $\mathrm{~S} 3$ & $\mathrm{~S} 4$ \\
\cline { 2 - 5 }$\left[10^{6} \mathrm{~m}^{3}\right]$ & 0.271 & 72.480 & 2.775 & 21.316 \\
\hline
\end{tabular}

These values have been used in order to evaluate a real economic response of the system: therefore, an economic post-processor has been constructed considering only the costs related to unplanned deficits and pumping operations. Herein, the unplanned deficit elements refer to the additional costs supported during shortage periods when demand requests can not be satisfied.

Table 2. Economic post-processor

\begin{tabular}{lccc}
\hline $\begin{array}{l}\text { Annual average } \\
\text { Costs }\end{array}$ & $\mathrm{C}_{\text {deficit }}$ & $\mathrm{C}_{\text {pumping }}$ & $\mathrm{C}_{\mathrm{TOT}}$ \\
\hline $10^{6} €$ /year & 0.277 & 2.578 & 2.855 \\
\hline
\end{tabular}

The costs reported in Table 2 are shown in terms of average annual values. Almost the total costs amount is due to energy contribution (pumping costs) while just a low contribution depends on the unplanned deficit occurrences, which means that almost the totality of system's users have been satisfied during the referring time horizon. These results highlight a huge reduction in terms of costs supported managing this real water system respect the current configuration, saving more than 1 million of euros. 


\subsection{Sensitivity analysis}

A sensitivity analysis has been performed in order to evaluate the dependences of costs on the volume thresholds used for defining the activation of pumps. For each pump station the resulting optimal fraction of volume $q_{p}$ can be obtained using the reservoir threshold values reported in Table 1 and relating these to the pumps by dependences to the connected reservoirs. The volume fractions are expressed in terms of values varying between 0 and 1 . Optimal values of volume fraction $q_{p} \in[0,1]$ are given in Table 3 .

Table 3. Optimal volume fractions

\begin{tabular}{lllll}
\hline Fractions & $\mathrm{q}_{1}$ & $\mathrm{q}_{2}$ & $\mathrm{q}_{3}$ & $\mathrm{q}_{4}$ \\
\cline { 2 - 5 } & 0.0201 & 0.1264 & 0.3061 & 0.0109 \\
\hline
\end{tabular}

In order to estimate the additional cost that can be expected if the management authority modifies the proposed optimal rule, an additional simulation process has been implemented varying, for each pump $p \in P$, the volume fraction $q_{p}$. During this process, the volume fractions for all other pumps will be kept constant and equal to the optimized values. The values of parameters for the selected pumps have been varied between theoretical lower and upper bounds 0 and 1 .
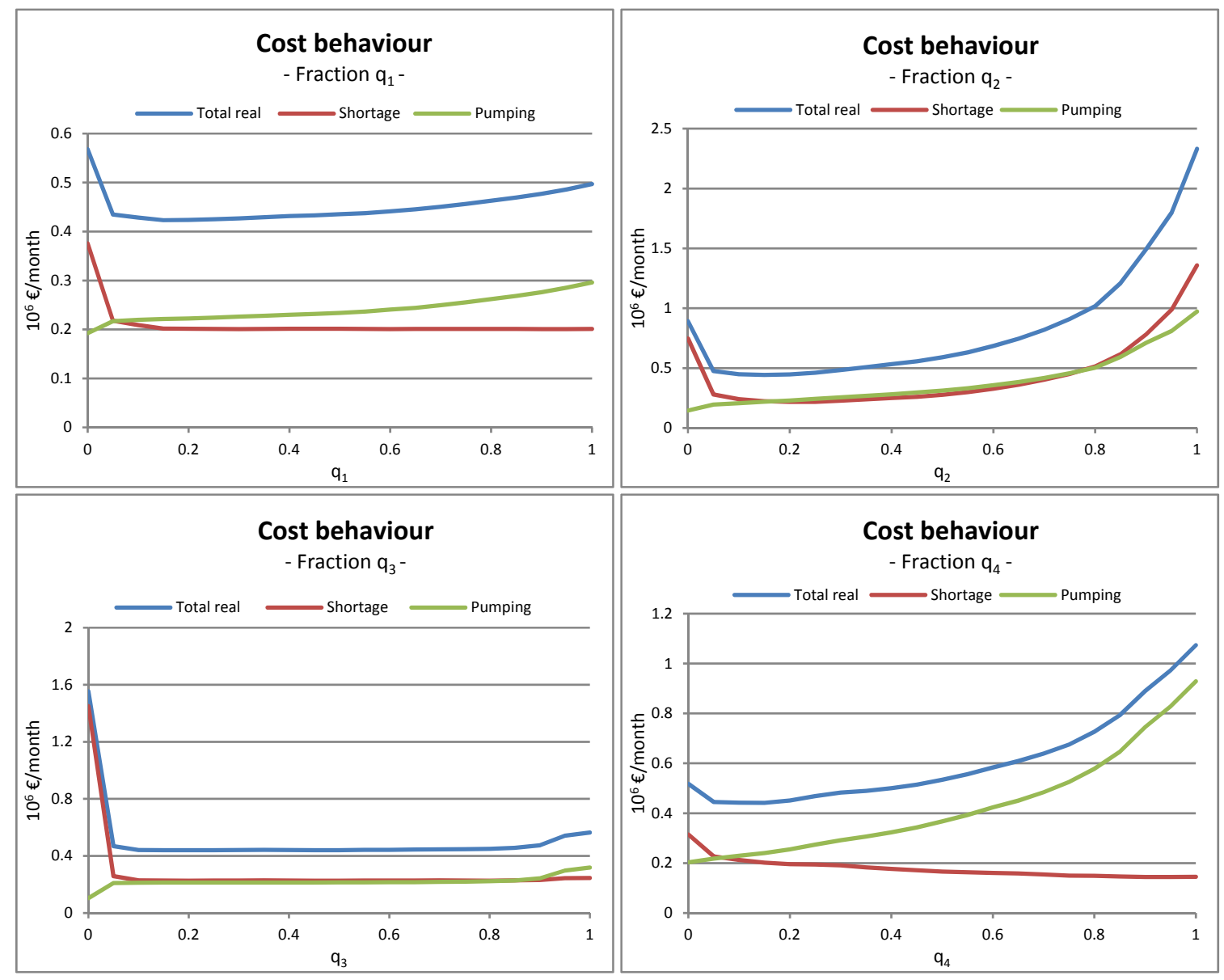

Figure 3. Real costs behaviour varying the pumping rules

The behaviour of costs is reported in Figure 3, where the blue line shows the dependence of the total costs on the changing parameter of pump activation rules. As shown before, these costs should be considered as the sum of two contributions: pumping costs (green line) and shortage costs (red line). The average monthly cost variations are reported in Figure 3. The total cost functions have the general tendency to increase smoothly with increasing volume fraction values, compared to the optimal ones. Instead, this increasing behaviour grow up much faster if we use a lower volume fraction compared to the optimal ones. This increasing behaviour is 
important and highlights a criticality if the authority should decide to use a lower volume fraction values for the activation of pumps.

Anyway, this behaviour can be easily explained: the increasing values are proportional to the costs of energy resulting from pumping and do not significantly deviate from a linear trend. Instead, the increasing values in the left part of the graph are related to the penalization of shortages that grow faster than linear. The anomalous behaviour of red line (shortage costs) for pump P2 is explained by the deficit occurrences in demands D2 if the water resource is diverted to other demands from reservoir R1.

\section{DISCUSSION AND CONCLUSIONS}

The approach reported in this paper aims to restrict the risks of deficit for the users and minimize the costs of managing the system in the conditions of water shortage. The recursive simulation and optimisation process based on the SQG method confirms its potential when applied to the water resource system management. This methodology allows to consider substantially large and complex models and to provide an enhancement for the optimal solutions under large uncertainty described by a significant number of synthetic scenarios. Moreover, by considering a long hydrological synthetically generated scenario, the SQG approach has managed to model such important features: the different resource availabilities and drought limitations in using resources, and the role of the water emergency links connected by pumping plants.

\section{REFERENCES}

Birge, J.R. and Louveaux, F. (2001). Introduction to Stochastic Programming, New York: Springer-Verlag.

Cunha M.C. and Sousa J.J.O. (2010). Robust design of water distribution networks for a proactive risk management. Journal of Water Resources Planning and Management, 136(2): 227-236.

Gaivoronski, A. (2005). SQG: Stochastic programming software environment. In S.W. Wallace and W.T. Ziemba, editors, Applications of Stochastic Programming. SIAM \& MPS; 4: 37-60.

Gaivoronski, A., Sechi, G.M. and Zuddas, P. (2012). Cost/risk balanced management of scarce resources using stochastic programming. European Journal of Operational Research; 216: 214-224.

Kang, D. and Lansey, K. (2014). Multiperiod Planning of Water Supply Infrastructure Based on Scenario Analysis. Journal of Water Resources Planning and Management; 140 (1): 40-54.

Napolitano, J., Sechi, G. M. and Zuddas, P. (2016). Scenario Optimization of Pumping Schedules in a Complex Water Supply System Considering a Cost-Risk Balancing Approach. Water Resource Management, 30: 5231-5246.

RAS. (2006). Piano Stralcio di Bacino Regionale per l’Utilizzo delle Risorse Idriche. Regione Autonoma della Sardegna, Italy.

Sechi, G.M. and Zuddas, P. (2008). Multiperiod hypergraph models for water systems optimization. Water Resource Management; 22: 307-320.

Yuan L., Zhou J., Li C., Xie M. and Mo L. (2016). Benefit and risk balance optimization for stochastic hydropower scheduling. Water Resource Management; 30: 3347-3361. 